\title{
SCHÄTZVERFAHREN FÜR DIE DISPOSITION VON PASSUNGSTEILEN
}

\author{
Thomas Christ, TH Karlsruhe
}

Passungsteile (Ausgleichsscheiben, Lamellen, Abstandsringe, ...) werden insbesondere zum Ausgleich von produktionsbedingten Toleranzen benötigt. Die Disposition dieser Teile bereitet Schwierigkeiten, da ihr Bedarf selbst bei determinierten Losgrößen von den Unregelmäßigkeiten des Produktionsprozesses abhängt, also stochastischer Natur ist. Das Problem tritt z.B. in der Motoren- und Getriebefertigung oder bei der Montage von Servolenkungen auf.

Anstatt den zukünftigen Bedarf mit Hilfe konventioneller Prognosemethoden (gleitende Mittelwerte, exponentielles Glätten, ...) aus den Vergangenheitsdaten zu ermitteln, wird hier versucht, aus den Verbrauchsdaten der Passungsteile die Funktionalparameter der Verteilung der zugrundeliegenden Störgröße zu schätzen. Dies erlaubt nicht nur eine Prognose des zukünftigen Bedarfs, sondern ermöglicht auch einen tieferen Einblick in die Stochastik des Fertigungsprozesses (Verteilung der Störgröße, zeitliche Veränderung, ...).

Um mit möglichst wenig verschiedenen Passungsteilen auszukommen, wird meist eine Kombination mehrerer Passungsteile gemäß einer definierten Einbaustrategie verwendet, wobei eine gewisse Resttoleranz verbleiberı darf. Geht man davon aus, daß die Störgröße nicht direkt gemessen werden kann, sondern aus den Verbrauchsdaten der Passungsteile geschätzt werden muß, dann erhält man ein Schätzproblem mit gruppierten Daten (aufgrund der Resttoleranz), bei dem die einzelnen Klassen - im Gegensatz zur herkömmlichen Problemstellung - nicht zusammenhängend und teilweise sogar überlappend sind (aufgrund der Kombinierbarkeit). Konventionelle Schätzverfahren können also nicht ohne weiteres verwendet werden.

Mittels Variationen der Maximum-Likelihood- und der $\chi^{2}$-Minimum-Methode sowie numerischer Approximationsverfahren (scoring system) wird eine Schätzung möglich. Ergebnisse aus einer Simulationsstudie mit verschiedenen Einbaustragien und Losgrößen zeigen, daß beide Verfahren bei großen Stichproben gute Resultate liefern. 